TRANSACTIONS OF THE

AMERICAN MATHEMATICAL SOCIETY

Volume 359, Number 6, June 2007, Pages 2777-2786

S 0002-9947(06)04077-3

Article electronically published on December 20, 2006

\title{
CLOSED MANIFOLDS COMING FROM ARTINIAN COMPLETE INTERSECTIONS
}

\author{
ŞTEFAN PAPADIMA AND LAURENŢIU PĂUNESCU
}

\begin{abstract}
We reformulate the integrality property of the Poincare inner product in the middle dimension, for an arbitrary Poincaré $\mathbb{Q}$-algebra, in classical terms (discriminant and local invariants). When the algebra is 1-connected, we show that this property is the only obstruction to realizing it by a smooth closed manifold, in dimension 8. We analyse the homogeneous artinian complete intersections over $\mathbb{Q}$ realized by smooth closed manifolds of dimension 8, and their signatures.
\end{abstract}

\section{INTRODUCTION}

1.1. Artinian complete intersection. Let $\mathcal{A}$ be a weighted artinian complete intersection $(W A C I)$, that is, a commutative graded $\mathbb{Q}$-algebra of the form

$$
\mathcal{A}=\mathbb{Q}\left[x_{1}, \ldots, x_{n}\right] / \mathcal{I}
$$

where the variables $x_{i}$ have positive even weights, $w_{i}:=\left|x_{i}\right|$, and the ideal $\mathcal{I}$ is generated by a regular sequence,

$$
\mathcal{I}=\left(f_{1}, \ldots, f_{n}\right),
$$

of weighted-homogeneous polynomials, $f_{i}$.

One knows [5, Theorem 3 and p. 198] that $\mathcal{A}^{*}$ is a 1-connected rational Poincaré duality algebra $(\mathbb{Q}-P D A)$, with Poincaré polynomial

$$
\mathcal{A}^{*}(t)=\prod_{i=1}^{n} \frac{1-t^{\left|f_{i}\right|}}{1-t^{\left|x_{i}\right|}}
$$

(and, consequently, with even formal dimension, $m=\sum_{i=1}^{n}\left(\left|f_{i}\right|-\left|x_{i}\right|\right)$ ).

1.2. The integrality obstruction. Let $\mathcal{A}^{*}$ be an arbitrary 1-connected Poincaré duality $\mathbb{Q}$-algebra, with formal dimension $m$. The smoothing problem we are going to look at is the following:

is $\mathcal{A}^{*}$ isomorphic to a graded algebra of the form $H^{*}\left(M^{m}, \mathbb{Q}\right)$, where

$M$ is a 1-connected closed smooth $m$-manifold?

Received by the editors July 26, 2004 and, in revised form, April 12, 2005

2000 Mathematics Subject Classification. Primary 57R65, 13C40; Secondary 11E81, 58K20.

Key words and phrases. $\mathbb{Q}$-surgery, artinian complete intersection, quadratic form, finite map germ.

The authors were partially supported by grant U4249 Sesqui R\&D/2003 of the University of Sydney.

(C)2006 American Mathematical Society 
We shall say that $\mathcal{A}$ is smoothable if the answer is yes.

By $\mathbb{Q}$-surgery ([1], 2]), we know that $\mathcal{A}$ is smoothable, for $m \neq 4 k$. Assume now that $m=4 k$, and pick an orientation, $\omega \in \mathcal{A}^{4 k} \backslash\{0\}$. This gives rise (via Poincaré duality) to a symmetric inner product space over $\mathbb{Q}$, denoted by $\left(\mathcal{A}^{2 k}, \cdot \omega\right) \in W(\mathbb{Q})$. (Here and in the sequel, $W(R)$ denotes the Witt group of the ring $R$; see [7.) If $\mathcal{A}$ is smoothable, then clearly

$$
\left(\mathcal{A}^{2 k}, \cdot \omega\right) \in W(\mathbb{Z}), \quad \text { for some orientation } \omega .
$$

It turns out that the integrality obstruction from (1.4), for a fixed orientation $\omega$, is equivalent to the fact that the quadratic form on $\mathcal{A}^{2 k}$ associated to $\cdot \omega$ is a sum of signed squares, over $\mathbb{Q}$; see [7, Corollary IV.2.6].

When the signature is zero, the integrality condition is equivalent to $\left(\mathcal{A}^{2 k}, \cdot\right)$ being split; see [7, I.6-7]. In this case, (1.4) is the only obstruction to smoothing; see [11] and 22, and also [9, Proposition 3.4]. In the non-zero signature case, additional obstructions may appear; see e.g. [9, §4.5] for some simple examples, based on [2] and [3].

1.3. Main results. The smoothing problem described above may be solved by using fundamental $\mathbb{Q}$-surgery results due to D. Sullivan; see [11, 2]. This opens the way for constructing closed manifolds with interesting geometric properties, starting from $\mathbb{Q}-P D A$ 's (see [9] for applications to geodesics).

The difficulties of the smoothing problem stem from the fact that the obstructions involve, besides (1.4), delicate conditions on the signature. In Section 2, we focus on the integrality obstruction. In Theorem 2.2, we show that (1.4) is the only obstruction to smoothing, for $\mathbb{Q}-P D A$ 's of formal dimension 8. (The same thing trivially holds true in dimension 4.) This is no longer true in dimension 12 ; see Remark 2.3. In dimension 8, our proof requires a classical result on sums of four squares.

As far as condition (1.4) is concerned, it may be handled, for a fixed orientation, by using discriminants and local invariants of non-degenerate quadratic forms over $\mathbb{Q}$; see [10]. We give a similar interpretation for (1.4), where $\mathcal{A}$ is an arbitrary $\mathbb{Q}-P D A$, in Theorem 2.5. by analysing changes of orientation. For odd rank, the answer depends only on local invariants. For even rank, both local invariants and discriminant are involved, in general; see Remark 2.6.

The results from Section 2 are applied in Section 3 . Here, we construct 8manifolds with interesting signatures, starting from WACI's which are homogeneous (that is, with $w_{i}=2$, for all $i$ ). The integrality test from Theorem 2.5 is illustrated on two families of examples: one with odd rank (see Example 3.3), and the other with even rank (see Example 3.5). The even rank family has the remarkable property that the corresponding test, described in Theorem 2.5)(2), collapses to a single, simple, discriminant obstruction.

1.4. Signature and degree. The signature plays an important role in the smoothing problem described in $\S 1.2$, via the Hirzebruch formula; see [8], 11, 2]. There is also an interesting connection with singularity theory, that seems worth mentioning at this point; see [4], 6], 1].

Let $\mathcal{A}=\mathbb{Q}\left[x_{1}, \ldots, x_{n}\right] /\left(f_{1}, \ldots, f_{n}\right)$ be an arbitrary $W A C I$, as defined in 1.1 Among other things, S. Halperin showed in [5, Theorem 3] that $\mathcal{A}^{*}$ is a Poincaré duality algebra (1-connected and commutative), thus giving rise to $(\mathcal{A}, \cdot \omega) \in W(\mathbb{Q})$, for any choice of orientation, $\omega \in \mathcal{A}^{m} \backslash\{0\}$. Let us consider the associated finite $C^{\infty}$ 
map germ, $f:\left(\mathbb{R}^{n}, 0\right) \rightarrow\left(\mathbb{R}^{n}, 0\right)$, having as components the defining polynomial relations of $\mathcal{A}$. Since $f^{-1}(0)=\{0\}$, the degree at 0 of $f, \operatorname{deg}(f)$, is defined, and may be computed in terms of regular values of $f$.

It follows from Theorem 1.2 of D. Eisenbud and H. Levine [4] (see also [1, pp. 103-104] and [6] that one has the following topological interpretation of the signature:

$$
\sigma(\mathcal{A}, \cdot \omega)=\operatorname{deg}(f)
$$

for a good choice of orientation, $\omega$. The above formula may be used in two ways. First, it gives an explicit way of computing the signature of $\mathcal{A}$ in terms of its defining relations (a question raised by Halperin [5, Section 9]). Second, it provides an algebraic recipe for computing topological degrees; see Remark 3.7 for some examples coming from homogeneous $W A C I$ 's.

\section{Smoothing in SMAll Dimensions, AND The Integrality CONDition}

2.1. Small dimensions. We begin by showing how the general smoothing problem becomes simpler, in small dimensions. In dimension 4 , clearly all 1 -connected $\mathbb{Q}-$ $P D A$ 's $\mathcal{A}$ satisfying (1.4) are smoothable, since

$$
\mathcal{A}^{*}=H^{*}\left(\left(\# \mathbb{C P}^{2}\right) \#\left(\#_{s} \overline{\mathbb{C P}^{2}}\right), \mathbb{Q}\right), \quad \text { or } \quad \mathcal{A}^{*}=H^{*}\left(S^{4}, \mathbb{Q}\right) \text {. }
$$

Our next result clarifies the first non-trivial case $(m=8)$.

Theorem 2.2. Let $\mathcal{A}$ be a 1-connected Poincaré duality $\mathbb{Q}$-algebra with formal dimension 8. Then $\mathcal{A}$ is smoothable (in the sense explained in \$1.2) if and only if there is $\omega \in \mathcal{A}^{8} \backslash\{0\}$ such that $\left(\mathcal{A}^{4}, \cdot \omega\right) \in W(\mathbb{Z})$.

Proof. We have to show that $\mathcal{A}$ is smoothable, as soon as property (1.4) from $₫ 1.2$ holds. Pick an orientation $\omega$ such that $\sigma:=\sigma\left(\mathcal{A}^{4},{ }_{\omega}\right) \geq 0$. We know that the Poincaré quadratic form on $\mathcal{A}^{4}$ is a sum of $t$ squares, $t \geq \sigma$, minus a sum of $s$ squares.

If $\sigma=0$, smoothability is guaranteed by (1.4); see $₫ 1.2$. Assume then that $\sigma>0$. We claim that if the system

$$
\begin{cases}a+b & =\sigma \\ 25 a+18 b & =\sum_{i=1}^{t} \alpha_{i}^{2}\end{cases}
$$

has integer solutions, then $\mathcal{A}$ is smoothable.

Indeed, we may take the following algebraic Pontrjagin classes: $q_{2}=(10 a+9 b) \omega$, and $q_{1}=\sum_{i=1}^{t} \alpha_{i} x_{i}$, where $\left\{x_{i}\right\}$ is the canonical basis of the positive definite part of $\mathcal{A}^{4}$. Set $N^{8}=a \cdot \mathbb{C P}^{4}+b \cdot \mathbb{C P}^{2} \times \mathbb{C P}^{2}$. Using the second equation from (2.2), one may easily check that $\mathcal{A}$ and $N$ have the same Pontrjagin numbers; see [8]. This implies, via the first equation from (2.2), that the Hirzebruch signature formula holds for $\mathcal{A}$, and we are done $([11,[2])$.

We come back to the system (2.2). If $t \geq 4$, the theorem of Bachet de MéziriacLagrange [7, II.8] guarantees integer solutions.

In the remaining cases, $\sigma$ must be 1,2 or 3, and then (2.2) may be solved as follows:

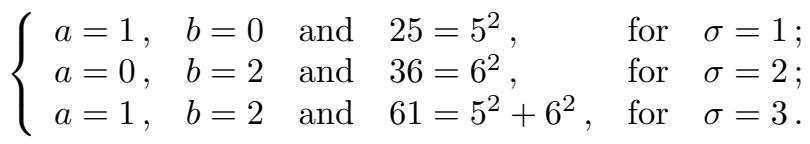

This completes our proof. 
Remark 2.3. The range $m \leq 11$ is the best one for which the integrality condition alone guarantees smoothability. Indeed, $\mathcal{A}=\mathbb{Q}[x] /\left(x^{3}\right)$, with $|x|=6$, has the integrality property, without being smoothable. See [9, §4.5].

2.4. An integrality test. In applications, we will need to check the integrality condition (1.4). This is clearly related to the theory of non-degenerate quadratic forms over $\mathbb{Q}$. We thus start by reviewing some relevant facts from 10 .

To begin with, assume that $\mathcal{A}$ is an arbitrary $\mathbb{Q}-P D A$, with formal dimension $4 k$. Set $r:=\operatorname{dim}_{\mathbb{Q}} \mathcal{A}^{2 k}$, and choose a $\mathbb{Q}$-basis of $\mathcal{A}^{2 k}$. Pick any orientation, $\omega \in$ $\mathcal{A}^{4 k} \backslash\{0\}$, and denote by $A_{\omega}$ the matrix of ${ }_{\omega}$. Note that $A_{\lambda \omega}=\lambda^{-1} \cdot A_{\omega}$, for any $\lambda \in \mathbb{Q}^{*}$. The condition $\left(\mathcal{A}^{2 k}, \cdot \omega\right) \in W(\mathbb{Z})$ translates to the fact that $A_{\omega}$ is equivalent over $\mathbb{Q}$ (in the classical sense, see [10, IV.1]) with a diagonal matrix of signs.

By a convenient choice of basis of $\mathcal{A}^{2 k}$, we may suppose that $A_{\omega}=\operatorname{diag}\left(a_{1}, \ldots, a_{r}\right)$. Then the discriminant of ${ }_{\omega} \omega$ is equal to $a_{1} \cdots a_{r}$ (modulo $\mathbb{Q}^{* 2}$ ). For each prime number $p$, one also has a local invariant at $p$, denoted by

$$
\varepsilon_{p}\left(A_{\omega}\right):=\prod_{1 \leq i<j \leq r}\left(a_{i}, a_{j}\right)_{p} \in\{ \pm 1\}
$$

where $(\cdot, \cdot)_{p}$ denotes the $p$-adic Hilbert symbol. From the classification theory $([10$, IV.3]), we infer that

$$
\left(\mathcal{A}^{2 k},{ }_{\omega}\right) \in W(\mathbb{Z}) \Longleftrightarrow\left|a_{1} \cdots a_{r}\right| \in \mathbb{Q}^{* 2} \quad \text { and } \quad \varepsilon_{p}\left(A_{\omega}\right)=1, \forall p \equiv 1(2) .
$$

Our next result translates in similar terms condition (1.4), by taking into account changes of orientation.

Theorem 2.5. Let $\mathcal{A}$ be an arbitrary $\mathbb{Q}-P D A$, of formal dimension $4 k$. Then:

(1) Assume $r \equiv 1(2)$. For any orientation $\omega$, there is $\lambda \in \mathbb{Q}^{*}$ such that the discriminant of $\cdot \lambda \omega$ is 1 (modulo $\mathbb{Q}^{* 2}$ ). Supposing that $\omega$ has the property that $a_{1} \cdots a_{r} \in \mathbb{Q}^{* 2}$, (1.4) is equivalent to

$$
\varepsilon_{p}\left(A_{\omega}\right)=1, \forall p \equiv 1(2) .
$$

(2) Assume $r \equiv 0(2)$. Let $\omega$ be an arbitrary orientation. Set $\epsilon:=\operatorname{sgn}\left(a_{1} \cdots a_{r}\right)$. Two cases may occur:

$(\bullet) r \equiv 0(4)$ and $\epsilon=+1$, or $r \equiv 2(4)$ and $\epsilon=-1$. In this case, (1.4) is equivalent to

$$
\left|a_{1} \cdots a_{r}\right| \in \mathbb{Q}^{* 2} \quad \text { and } \quad \varepsilon_{p}\left(A_{\omega}\right)=1, \forall p \equiv 1(2) .
$$

$(\bullet \bullet) r \equiv 0$ (4) and $\epsilon=-1$, or $r \equiv 2(4)$ and $\epsilon=+1$. In this case, (1.4) is equivalent to

$$
\left|a_{1} \cdots a_{r}\right| \in \mathbb{Q}^{* 2} \quad \text { and } \quad \varepsilon_{p}\left(A_{\omega}\right)=1, \forall p \equiv 1(4) .
$$

Proof. Part (11). The first assertion is easy: one may take for instance $\lambda=a_{1} \cdots a_{r}$. As for the second one, it will follow from (2.3), as soon as the following claim is proved: if $\left(\mathcal{A}^{2 k}, \cdot{ }^{2} \omega\right) \in W(\mathbb{Z})$, where $\lambda>0$, then $\left(\mathcal{A}^{2 k}, \cdot \omega\right) \in W(\mathbb{Z})$. To verify this claim, note that the first condition in $(2.3)$ implies that necessarily $\lambda \in \mathbb{Q}^{* 2}$; this in turn ensures that $\varepsilon_{p}\left(\lambda^{-1} A_{\omega}\right)=\varepsilon_{p}\left(A_{\omega}\right)$, for all $p$ (since Hilbert symbols are well-defined modulo squares; see [10, III.1]), and we are done.

Part (2). If $r$ is even, it readily follows from (2.3) that (1.4) is equivalent to the fact that there is $\lambda \in \mathbb{Q}^{*}, \lambda>0$, having the property that

$$
\left|a_{1} \cdots a_{r}\right| \in \mathbb{Q}^{* 2} \text { and } \varepsilon_{p}\left(\lambda A_{\omega}\right)=1, \forall p \equiv 1(2) .
$$


It remains to compute the local invariants of $\lambda \cdot A_{\omega}$, at odd primes. This may be done as follows. First, one may use the bilinearity of Hilbert symbols ([10, III.1]), together with the first property from (2.4), to see that

$$
\varepsilon_{p}\left(\lambda A_{\omega}\right)=\varepsilon_{p}\left(A_{\omega}\right) \cdot(\lambda, \lambda)^{\frac{r(r-1)}{2}} \cdot(\lambda, \epsilon)_{p} .
$$

(•) In this case, elementary properties of Hilbert symbols ([10, III.1]) imply that (2.5) above reduces to

$$
\varepsilon_{p}\left(\lambda A_{\omega}\right)=\varepsilon_{p}\left(A_{\omega}\right)
$$

and we are done.

(••) Similarly, in this case (2.5) becomes

$$
\varepsilon_{p}\left(\lambda A_{\omega}\right)=\varepsilon_{p}\left(A_{\omega}\right) \cdot(\lambda, \lambda)_{p} .
$$

Note that $(\mu \nu, \mu \nu)_{p}=(\mu, \mu)_{p}(\nu, \nu)_{p}$ and $(2,2)_{p}=1$ (10, III.1]). It follows that we may assume in (2.4) that $\lambda$ is a product of distinct odd primes, $\lambda=q_{1} \cdots q_{l}$. Use [10, III.1] to compute

$$
(\lambda, \lambda)_{p}=\prod_{i=1}^{l}\left(q_{i}, q_{i}\right)_{p}= \begin{cases}1, & \text { for } p \neq q_{1}, \ldots, q_{l} ; \\ (-1)^{\varepsilon\left(q_{j}\right)}, & \text { for } p=q_{j},\end{cases}
$$

where $\varepsilon(q)$ denotes the residue class modulo 2 of $\frac{q-1}{2}$, as in 10. We infer from (2.4), (2.7) and (2.8) that (1.4) implies the conditions from our statement.

Conversely, set

$$
\left\{p=\text { odd } \mid \varepsilon_{p}\left(A_{\omega}\right)=-1\right\}=\left\{q_{1}, \ldots, q_{l}\right\} .
$$

If all primes $q_{j}$ appearing in (2.9) above are equal to 3 (modulo 4), then we may take $\lambda=q_{1} \cdots q_{l}$, and (1.4) follows, again from (2.4), (2.7), and (2.8). Our proof is complete.

Remark 2.6. Let $(V, \cdot)$ be a symmetric inner product space over $\mathbb{Q}$ (alias, a nondegenerate quadratic $\mathbb{Q}$-form). For any $k,(V, \cdot)$ may obviously be realized as $\left(\mathcal{A}^{2 k}, \cdot \omega\right)$, where the oriented $\mathbb{Q}-P D A \mathcal{A}^{*}$ is $\mathbb{Q} \cdot 1$, in degree $*=0, V$ in degree $*=2 k, \mathbb{Q} \cdot \omega$ in degree $*=4 k$, and 0 otherwise, with product given by $\cdot$

Note first that the condition $\left(\mathcal{A}^{2 k}, \cdot \lambda \omega\right) \in W(\mathbb{Z})$ may depend on $\lambda \in \mathbb{Q}^{*}$. For odd $r$, examples are easy to construct, using the discriminant obstruction from (2.3). For $r=2$, for instance, a simple example is provided by the quadratic form with matrix $A=\operatorname{diag}(5,5)$. Here, $\left(\mathcal{A}^{2 k}, \cdot \omega\right) \in W(\mathbb{Z})$, while $\left(\mathcal{A}^{2 k}, \cdot_{3 \omega}\right) \notin W(\mathbb{Z})$, even though the discriminant condition from (2.3) is verified.

Note also that, in general, the local invariants show up in an essential way, in our integrality test from Theorem 2.5 (2). Indeed, consider the matrix $A=$ $\operatorname{diag}(1,1,1,2,5,10)$. The associated $\mathbb{Q}-P D A$ belongs to case $(\bullet \bullet)$, and satisfies the discriminant condition therefrom. On the other hand, $\varepsilon_{5}(A)=-1$, as readily seen.

\section{Homogeneous COMPLETE intersections}

In this section, we want to apply Theorem 2.2 to $W A C I$ 's. We will restrict our attention to homogeneous $W A C I$ 's, i.e., those with $w_{i}=2$ and $\left|f_{i}\right|=2 d_{i} \geq 4$, for all $i$. Both conditions are very natural. The first one simply means that each $f_{i}$ is a homogeneous polynomial of degree $d_{i}$. The restrictions $d_{i} \geq 2(1 \leq i \leq n)$ are imposed to avoid unnecessary redundancies, like $\mathbb{Q}[x] /(x)=\mathbb{Q}$. 
It is straightforward to check that the formal dimension is equal to 8 precisely in the cases listed below (where $\underline{d}$ denotes $\left(d_{1}, \ldots, d_{n}\right)$, and $\left.r:=\operatorname{dim}_{\mathbb{Q}} \mathcal{A}^{4}\right)$; see $(1.3)$.

$$
\begin{aligned}
& \left(I_{8}\right) \quad \underline{d}=(2,2,2,2) ; \quad r=6 . \\
& \left(I I_{8}\right) \quad \underline{d}=(2,2,3) ; \quad r=4 \text {. } \\
& \left(I I I_{8}\right) \quad \underline{d}=(2,4) ; \quad r=2 \text {. } \\
& \left(I V_{8}\right) \quad \underline{d}=(3,3) ; \quad r=3 \text {. } \\
& \left(V_{8}\right) \quad \underline{d}=(5) ; \quad r=1 .
\end{aligned}
$$

Note that, in the general homogeneous $W A C I$ case, every degree vector, $\underline{d}$, may be realized by a smooth manifold. Indeed, $H^{*}\left(\prod_{i=1}^{n} \mathbb{C} \mathbb{P}^{d_{i}-1}, \mathbb{Q}\right)=\bigotimes_{i=1}^{n} \mathbb{Q}\left[x_{i}\right] /\left(x_{i}^{d_{i}}\right)$, with signature 1 , when all $d_{i}$ 's are odd, and 0 , otherwise. One may ask whether more interesting signatures may also arise from smooth manifolds. For instance, in case $\left(I_{8}\right)$ above, the possible (non-negative) values of the signature are $0,2,4,6$ (since $r=6$ ). Our last main result completely clarifies this question.

Theorem 3.1. All possible values of $\underline{d}$ and of the signature of homogeneous $W A C I$ 's with formal dimension $m=8$ may be realized by smooth manifolds.

In the next lemma, we take care of the subcases where the desired manifold may be obtained from known examples, by taking products and connected sums.

Lemma 3.2. Theorem 3.1 is true, in all cases different from $\left(I V_{8}\right), \sigma=3$, and $\left(I_{8}\right), \sigma=2$ or 6 .

Proof. If $\sigma=0$ or 1 , one may use products of complex projective spaces, as explained before.

Case $\left(I_{8}\right), \sigma=4: H^{*}\left(\left(\mathbb{C P}^{2} \# \mathbb{C P}^{2}\right) \times\left(\mathbb{C P}^{2} \# \mathbb{C P}^{2}\right), \mathbb{Q}\right)$ is equal to

$$
\mathbb{Q}\left[x_{1}, x_{2}, y_{1}, y_{2}\right] /\left(x_{1}^{2}-x_{2}^{2}, x_{1} x_{2}, y_{1}^{2}-y_{2}^{2}, y_{1} y_{2}\right) \text {. }
$$

Case $\left(I I_{8}\right), \sigma=2: H^{*}\left(\left(\mathbb{C P}^{2} \# \mathbb{C P}^{2}\right) \times \mathbb{C P}^{2}, \mathbb{Q}\right)=\mathbb{Q}\left[x_{1}, x_{2}, x_{3}\right] /\left(x_{1}^{2}-x_{2}^{2}, x_{1} x_{2}, x_{3}^{3}\right)$.

Case $\left(I I_{8}\right), \sigma=4: \mathcal{A}=\mathbb{Q}\left[x_{1}, x_{2}, x_{3}\right] /\left(x_{1}^{2}-x_{3}^{2}, x_{2}^{2}-x_{3}^{2}, x_{1} x_{2} x_{3}\right)$ is a smoothable homogeneous $W A C I$, with signature 4; see [9, Proposition 4.6].

Case $\left(I I I_{8}\right), \sigma=2: H^{*}\left(\mathbb{C P}^{4} \# \mathbb{C P}^{4}, \mathbb{Q}\right)=\mathbb{Q}\left[x_{1}, x_{2}\right] /\left(x_{1}^{4}-x_{2}^{4}, x_{1} x_{2}\right)$.

To check the remaining cases, we will use the integrality test from Theorem 2.5 . Case $\left(I V_{8}\right)$ will follow from the analysis of the family below.

Example 3.3. Let $\mathcal{A}(c), c \in \mathbb{Q}$, be the graded algebra

$$
\mathbb{Q}[x, y] /\left(f_{1}=x^{3}-x y^{2}, f_{2}=y^{3}-c x^{2} y\right),
$$

with $x$ and $y$ of degree 2 .

It is immediate to see that $\mathcal{A}(c)$ is a $W A C I$ (homogeneous, belonging to case $\left.\left(I V_{8}\right)\right)$ precisely when $\left\{f_{1}=f_{2}=0\right\}=\{0\}$ (over $\mathbb{C}$ ), that is, if and only if $c \neq 1$.

The next lemma completes the proof of Theorem 3.1 case $\left(I V_{8}\right)$, and illustrates the arithmetic behind integrality condition (1.4).

Lemma 3.4. Let $\{\mathcal{A}(c)\}_{c \neq 1}$ be the above $W A C I$ family. Then:

(1) The absolute value of the signature of $\mathcal{A}(c)$ is $2+\epsilon$, where $\epsilon=\operatorname{sgn}(c-1)$.

(2) $\mathcal{A}(c)$ is smoothable $\Longleftrightarrow|c-1|$ is a sum of two rational squares. 
Proof. Part (11). It is readily checked that the matrix of the Poincaré quadratic form on $\mathcal{A}^{4}(c)$, with respect to the basis $\left\{x y, x^{2}, x^{2}-y^{2}\right\}$ and the orientation $\omega=(c-1) x^{4}$, is $A(c)=\operatorname{diag}\left(\frac{1}{c-1}, \frac{1}{c-1}, 1\right)$. Clearly, the signature of $\mathcal{A}(c)$ is as asserted.

Part (2). To decide the smoothability of $\mathcal{A}(c)$, we will use Theorem 2.5(1). Obviously, the orientation $\omega$ satisfies the required discriminant property. Therefore, $\mathcal{A}(c)$ is smoothable if and only if $\left(\frac{1}{c-1}, \frac{1}{c-1}\right)_{p}=1$, at all odd primes. This is equivalent $\left(\left[10\right.\right.$, III.1]) to $\left(\frac{1}{\epsilon(c-1)}, \frac{1}{\epsilon(c-1)}\right)_{p}=1$, at all odd primes and also at $\infty$. By Hilbert's theorem (see [10, III.2]), this is further equivalent to $\left(\frac{1}{\epsilon(c-1)}, \frac{1}{\epsilon(c-1)}\right)_{p}=1$, at all primes and also at $\infty$.

The definition of Hilbert symbols ([10, III.1]) and the Hasse-Minkowski theorem ([10, IV.3]) together imply that this happens if and only if $\epsilon(c-1)$ is a sum of two rational squares. The proof of part (2) is complete.

The last case of Theorem $3.1\left(\left(I_{8}\right), \sigma=2\right.$ or 6$)$ will be covered by analysing a second family.

Example 3.5. Let us consider the family of graded algebras $\{\mathcal{B}(c)\}_{c \in \mathbb{Q}}$, with weight 2 generators, $\left\{x_{i}\right\}_{1 \leq i \leq 4}$, and defining relations

$$
\left\{\begin{array}{l}
x_{i}^{2}-x_{4}^{2}, \quad \text { for } \quad i \leq 3 \\
\sum_{1 \leq i<j \leq 4} x_{i} x_{j}-c x_{4}^{2} .
\end{array}\right.
$$

It is easy to see that (3.1) defines a $W A C I$ (homogeneous, belonging to case $\left.\left(I_{8}\right)\right)$ if and only if $c \neq-2,0,6$. For $c=-1$, (3.1) defines the signature 6 algebra from [4, p. 24] (which is not smoothable, by Lemma 3.6 below).

The next lemma completes the proof of Theorem 3.1. For its proof, we will resort to the integrality test from Theorem 2.5)(2). At this point, it seems worthwhile pointing out that part (2) of the lemma provides an interesting family of examples, where property (1.4) may be decided using only the (simple) discriminant obstruction. This simple behaviour cannot be expected, in general; see Remark 2.6.

Lemma 3.6. Let $\{\mathcal{B}(c)\}_{c \neq-2,0,6}$ be the above $W A C I$ family. Then:

(1) The signature of $\mathcal{B}(c)$ is $0, \pm 2$ or \pm 6 .

(2) $\mathcal{B}(c)$ is smoothable if and only if $|(c-6)(c+2)| \in \mathbb{Q}^{* 2}$.

(3) For $c=-3,2$ and $-\frac{2}{5}$, the algebra $\mathcal{B}(c)$ is smoothable, with signature 0,2 and 6 , respectively.

Proof. Part (1). Our first task is to find a $\mathbb{Q}$-basis of $\mathcal{B}^{4}(c)$, and an orientation $\omega$, with respect to which the matrix of the Poincaré inner product is diagonal. We will begin with the basis $\left\{x_{i} x_{j}\right\}_{1 \leq i<j \leq 4}$. Set $y:=x_{i}^{2} \in \mathcal{B}^{4}(c), 1 \leq i \leq 4$. We claim that

$$
x_{1} x_{2} x_{3} x_{4}=\frac{c^{2}-4 c-6}{6} y^{2}
$$

and

$$
y x_{i} x_{j}=\frac{c}{6} y^{2}, \quad \text { for } \quad 1 \leq i<j \leq 4,
$$

so we may take $\omega=y^{2}$. Indeed, we infer from (3.1) that

$$
c y x_{1} x_{2}=c y x_{3} x_{4}=y^{2}+y\left(x_{1}+x_{2}\right)\left(x_{3}+x_{4}\right)+x_{1} x_{2} x_{3} x_{4} .
$$

Adding all relations of type (3.4), we get (3.2). Using (3.2), (3.3) follows from (3.4). 
Now consider the following basis in the middle dimension 4: $e_{1}=x_{1} x_{2}-$ $x_{3} x_{4}, e_{2}=x_{1} x_{4}-x_{3} x_{2}, e_{3}=x_{1} x_{3}-x_{2} x_{4}, e_{4}=x_{1} x_{2}+x_{3} x_{4}, e_{5}=x_{1} x_{4}+x_{3} x_{2}, e_{6}=$ $x_{1} x_{3}+x_{2} x_{4}$, and rescale the orientation to $\frac{(6-c)(c+2)}{3} y^{2}$.

With respect to these data, the intersection form is given by the following matrix:

$$
B_{1}(c)=\left(\begin{array}{cccccc}
1 & 0 & 0 & 0 & 0 & 0 \\
0 & 1 & 0 & 0 & 0 & 0 \\
0 & 0 & 1 & 0 & 0 & 0 \\
0 & 0 & 0 & a & b & b \\
0 & 0 & 0 & b & a & b \\
0 & 0 & 0 & b & b & a
\end{array}\right)
$$

where $a=\frac{c(c-4)}{(6-c)(c+2)}, b=\frac{2 c}{(6-c)(c+2)}$; use (3.2) and (3.3).

First note that if $c=4$, i.e. $a=0$, the determinant of $B_{1}$ is positive, so clearly this case cannot produce $\sigma=4$.

Let us now consider the case $c \neq 4$, i.e. $a \neq 0$.

Considering a new basis, $f_{i}=e_{i}, i \leq 4, f_{5}=e_{5}-e_{6}, f_{6}=-2 b e_{4}+a e_{5}+a e_{6}$, we obtain a new matrix:

$$
B_{2}(c)=\left(\begin{array}{cccccc}
1 & 0 & 0 & 0 & 0 & 0 \\
0 & 1 & 0 & 0 & 0 & 0 \\
0 & 0 & 1 & 0 & 0 & 0 \\
0 & 0 & 0 & a & 0 & 0 \\
0 & 0 & 0 & 0 & 2(a-b) & 0 \\
0 & 0 & 0 & 0 & 0 & 2 a(a-b)(a+2 b)
\end{array}\right) .
$$

Rescaling our last basis to $g_{i}=f_{i}, i \leq 5, g_{6}=\frac{(6-c)(c+2)}{c^{2}} f_{6}$, we finally obtain the matrix

$$
B_{3}(c)=\left(\begin{array}{cccccc}
1 & 0 & 0 & 0 & 0 & 0 \\
0 & 1 & 0 & 0 & 0 & 0 \\
0 & 0 & 1 & 0 & 0 & 0 \\
0 & 0 & 0 & \frac{c(c-4)}{(6-c)(c+2)} & 0 & 0 \\
0 & 0 & 0 & 0 & \frac{-2 c}{c+2} & 0 \\
0 & 0 & 0 & 0 & 0 & \frac{-2(c-4)}{c+2}
\end{array}\right)
$$

Our assertion on signature from part (1) easily follows by examining the distribution of signs on the diagonal of the matrix $B_{3}(c)$.

Part (3). Follows from part (2).

Part (2). The algebra $\mathcal{B}(c)$ is smoothable if and only if it verifies the integrality test from Theorem 2.5](2). The discriminant may be computed as $\operatorname{det} B_{1}(c) \equiv$ $(6-c)(c+2)$ (modulo $\left.\mathbb{Q}^{* 2}\right)$. This shows that we may assume from now on $c \neq 4$, and use the matrix $B_{3}(c)$. Set $\epsilon:=\operatorname{sgn}((6-c)(c+2))$, and note that $\epsilon=+1$ (respectively $\epsilon=-1$ ) corresponds to the case $(\bullet \bullet)$ (respectively $(\bullet)$ ). We have to show that, in both cases, the property $|(6-c)(c+2)| \in \mathbb{Q}^{* 2}$ implies the restrictions on the local invariants of $B:=B_{3}(c)$ from Theorem 2.5. Set

$$
\lambda_{1}=\frac{-2 c}{c+2}, \quad \lambda_{2}=\frac{-2(c-4)}{c+2}, \quad \text { and } \quad \lambda_{3}=\frac{c(c-4)}{(6-c)(c+2)}
$$


and note that $\lambda_{3} \equiv \epsilon \lambda_{1} \lambda_{2}$ (modulo $\mathbb{Q}^{* 2}$ ), by our assumption on the discriminant. By elementary manipulations with Hilbert symbols, we infer that

$$
\varepsilon_{p}(B)= \begin{cases}\left(\lambda_{1}, \lambda_{2}\right)_{p}, & \text { if } \epsilon=-1 ; \\ \left(\lambda_{1}, \lambda_{2}\right)_{p} \cdot\left(\lambda_{1}, \lambda_{1}\right)_{p} \cdot\left(\lambda_{2}, \lambda_{2}\right)_{p}, & \text { if } \epsilon=+1 .\end{cases}
$$

The discriminant condition means that

$$
\epsilon(6-c)=\frac{t^{2}}{s^{2}}(c+2),
$$

where $t$ and $s$ are relatively prime integers. Solve for $c$ and substitute in (3.6) to obtain the following values (modulo $\mathbb{Q}^{* 2}$ ) for $\lambda_{1,2}$ :

$$
\left\{\begin{array}{l}
\lambda_{1}=2 \epsilon t^{2}-6 s^{2} \\
\lambda_{2}=6 \epsilon t^{2}-2 s^{2}
\end{array}\right.
$$

We are going to compute the Hilbert symbols appearing in (3.7), in terms of Legendre symbols; see [10, I.3 and Theorem III.1]. To do this, write

$$
\left\{\begin{array}{l}
\lambda_{1}=p^{\alpha} u \\
\lambda_{2}=p^{\beta} v
\end{array}\right.
$$

where $\alpha, \beta \in \mathbb{N}, u, v \in \mathbb{Z}$, and $u, v \not \equiv 0(p)$.

To finish our proof, we are going to show that $\varepsilon_{p}(B)=1, \forall p \equiv 1(2)$ (when $\epsilon=-1)$, and $\varepsilon_{p}(B)=1, \forall p \equiv 1(4)($ when $\epsilon=+1)$.

Several cases may appear in (3.9). If $\alpha=\beta=0$, then plainly $\left(\lambda_{1}, \lambda_{2}\right)_{p}=$ $\left(\lambda_{1}, \lambda_{1}\right)_{p}=\left(\lambda_{2}, \lambda_{2}\right)_{p}=1$, at all odd primes $p$. The case $\alpha, \beta>0$ cannot occur, since this would imply (see (3.8)) that $s \equiv t \equiv 0(p)$. The remaining cases $(\alpha=0, \beta>0$ and $\alpha>0, \beta=0$ ) may be settled as follows.

For $\alpha=0, \beta>0$, one knows ([10, Theorem III.1]) that $\left(\lambda_{1}, \lambda_{2}\right)_{p}=\left(\frac{\lambda_{1}}{p}\right)^{\beta}$. Since $2 s^{2} \equiv 6 \epsilon t^{2}(p), \lambda_{1} \equiv-16 \epsilon t^{2}(p)$. Therefore, $\left(\lambda_{1}, \lambda_{2}\right)_{p}=(-\epsilon)^{\beta \varepsilon(p)}$. Similarly, for $\alpha>0, \beta=0$, one has $\left(\lambda_{1}, \lambda_{2}\right)_{p}=\left(\frac{\lambda_{2}}{p}\right)^{\alpha}$, with $\lambda_{2} \equiv 16 s^{2}(p)$, hence $\left(\lambda_{1}, \lambda_{2}\right)_{p}=1$. By (3.7), this completes our proof, when $\epsilon=-1$.

Assume now $\epsilon=+1$. In this last case, we will also need $\left(\lambda_{1}, \lambda_{1}\right)_{p}=(-1)^{\alpha \varepsilon(p)}$, and $\left(\lambda_{2}, \lambda_{2}\right)_{p}=(-1)^{\beta \varepsilon(p)}$. When $p \equiv 1(4)$, both $\left(\lambda_{1}, \lambda_{1}\right)_{p}$ and $\left(\lambda_{2}, \lambda_{2}\right)_{p}$ are 1 , which completes our proof (see (3.7)).

Remark 3.7. Let $f:\left(\mathbb{R}^{n}, 0\right) \rightarrow\left(\mathbb{R}^{n}, 0\right)$ be a finite map germ, whose components are homogeneous $\mathbb{R}$-polynomials, with degree vector $\underline{d}$, where $d_{i} \geq 2$, for all $i$. Assume that the degree of the jacobian, $J(f)=\operatorname{det}\left(\partial f_{i} / \partial x_{j}\right)$, is 4 . Set $\delta=|\operatorname{deg}(f)|$. Via Theorem 1.2 of [4], our Theorem 3.1 may be reinterpreted as describing all absolute values of topological degrees, for all possible values of $\underline{d}$ (as listed at the beginning of this section):

$$
\begin{array}{lll}
\left(I_{8}\right) & \underline{d}=(2,2,2,2) ; & \delta=0,2,4,6 . \\
\left(I I_{8}\right) & \underline{d}=(2,2,3) ; & \delta=0,2,4 . \\
\left(I I I_{8}\right) & \underline{d}=(2,4) ; & \delta=0,2 . \\
\left(I V_{8}\right) & \underline{d}=(3,3) ; & \delta=1,3 . \\
\left(V_{8}\right) & \underline{d}=(5) ; & \delta=1 .
\end{array}
$$


Remark 3.8. In dimension 4, obviously all signatures may be realized by 1-connected smooth closed manifolds; see (2.1). On the other hand, it is known that $\mathcal{A}^{*}=$ $H^{*}\left(M^{4}, \mathbb{Q}\right)$ is a $W A C I$ if and only if $b_{2}\left(M^{4}\right) \leq 2$; see for instance [12, p. 427]. In other words, this means that either $\mathcal{A}^{*}=H^{*}\left(S^{4}, \mathbb{Q}\right)$ or $s+t \leq 2$. (In the second case, note how homogeneous $W A C I$ 's naturally occur in dimension 4.) Plainly, $|\sigma|=0,1$ or 2 .

\section{REFERENCES}

[1] Arnold, V. I., Gusein-Zade, S. M., Varchenko, A. N.: Singularities of differentiable maps, Volume I. Monographs in Math., vol. 82, Boston-Basel-Stuttgart: Birkhäuser 1985. MR0777682 (86f:58018)

[2] Barge, J.: Structures différentiables sur les types d'homotopie rationnelle simplement connexes, Ann. Scient. Éc. Norm. Sup. 4e série, t. 9, 469-501 (1976). MR0440574 (55:13448)

[3] Barge, J., Lannes, J., Latour, F., Vogel, P.: $\Lambda$-sphères, Ann. Scient. Éc. Norm. Sup. 4e série, t. 7, 463-506 (1974). MR0377939 (51:14108)

[4] Eisenbud, D., Levine, H. I.: An algebraic formula for the degree of a $C^{\infty}$ map germ, Ann. Math. 106, 19-44 (1977). MR0467800 (57:7651)

[5] Halperin, S.: Finiteness in the minimal models of Sullivan, Trans. Amer. Math. Soc. 230, 173-199 (1977). MR0461508 (57:1493)

[6] Khimshiashvili, G. N.: On the local degree of a smooth map, Comm. Acad. Sci. Georgian SSR 85, 309-311 (1977). MR0458467 (56:16670)

[7] Milnor, J. W., Husemoller, D.: Symmetric bilinear forms. Ergebnisse der Math., vol. 73, Berlin-Heidelberg-New York: Springer 1973. MR0506372 (58:22129)

[8] Milnor, J. W., Stasheff, J. D.: Characteristic classes. Annals of Math. Studies, vol. 76, Princeton: Princeton Univ. Press 1974. MR0440554 (55:13428)

[9] Papadima, S., Paunescu, L.: Isometry-invariant geodesics and nonpositive derivations of the cohomology, J. Differential Geom. 71 159-176 (2005). MR2191771

[10] Serre, J.-P.: A course in arithmetic. Grad. Texts in Math., vol. 7, Berlin-Heidelberg-New York: Springer-Verlag 1996. MR0344216 (49:8956)

[11] Sullivan, D.: Infinitesimal computations in topology, Publ. Math. IHES 47, 269-331 (1977). MR0646078 (58:31119)

[12] Vigué-Poirrier, M.: Homotopie rationnelle et croissance du nombre de géodésiques fermées, Ann. Scient. Éc. Norm. Sup. 4e série, t. 17, 413-431 (1984). MR0777376 (86h:58027)

Institute of Mathematics "Simion Stollow", P.O. Box 1-764, RO-014700 Bucharest, ROMANIA

E-mail address: Stefan.Papadima@imar.ro

School of Mathematics and Statistics, University of Sydney, Sydney, New South Wales 2006, Australia

E-mail address: laurent@maths.usyd.edu.au 